\title{
EXTRINSIC CURVATURE OF SEMICONVEX SUBSPACES IN ALEXANDROV GEOMETRY
}

\author{
STEPHANIE B. ALEXANDER AND RICHARD L. BISHOP
}

\begin{abstract}
In Alexandrov spaces of curvature bounded either above (CBA) or below (CBB), we obtain extrinsic curvature bounds on subspaces associated with semiconcave functions. For CBA spaces, we obtain new intrinsic curvature bounds on subspaces. For CBB spaces whose boundary is extrinsically curved, we strengthen Perelman's concavity theorem for distance from the boundary, deriving corollaries on sharp diameter bounds, contractibility, and rigidity.
\end{abstract}

\section{Contents}

1. Introduction

2. Almost concave functions 6

3. Very concave functions $\quad 12$

4. Curved boundaries 15

5. Base-angle/chord and arc/chord curvature bounds 20

Acknowledgments $\quad 22$

References $\quad 22$

\section{INTRODUCTION}

Semiconcave functions arise naturally in Alexandrov geometry, and are powerful tools for studying the underlying spaces [AB2, Pt2, KPT]. Here we establish extrinsic curvature bounds on subspaces associated with semiconcave functions, in spaces of curvature bounded either above (CBA) or below (CBB). Recall that the superlevels of concave functions are convex. In the CBA and CBB settings, we use gradient curve techniques to derive upper bounds on the degree of nonconvexity of the superlevels of almost concave functions. Similarly, we derive lower bounds on the degree of convexity of level sets of very concave functions. For CBA spaces, new intrinsic curvature bounds on subspaces follow. For CBB spaces whose boundaries have strong extrinsic curvature bounds below, we give the correct strengthening of Perelman's theorem on concavity of distance from the boundary. Specifically, we replace the concavity inequality, which may be regarded as pushing the interiors of geodesics away from the boundary $\partial X$, by a stronger differential inequality that may be regarded as pulling them toward a "ghost center" of $X$. There are corollaries on sharp diameter bounds, contractibility, and rigidity.

1991 Mathematics Subject Classification. 53C20, 53C70, 53B25, 58C05.

Key words and phrases. Alexandrov spaces, CAT(K) spaces, semi-concave functions, subspaces, extrinsic curvature. 
1.1. Extrinsic curvature bounds. Throughout the paper (with the exception of Section 4, where $X$ is a finite-dimensional CBB space), $X$ will be a complete geodesic metric space, not necessarily locally compact, either CBB or CBA by $K$ (see definitions below). As general references, see [Ba, BN, BH, BBI, Pl, Pt2].

Definition 1.1. A subset $Y$ of $X$ has extrinsic curvature $\leq A$ if there is a function $F(r) \geq 0$ satisfying $\lim _{r \rightarrow 0}\left(F(r) / r^{3}\right)=0$, such that for every pair of points with distance $s<s_{0}$ in $Y$ and distance $r$ in $X$,

$$
s-r \leq\left(A^{2} / 24\right) r^{3}+F(r) .
$$

If we only consider pairs of points in an open subset $Z \subset Y$, then $Y$ has extrinsic curvature $\leq A$ on $Z$.

Remark 1.2. A chord of $Y$ is a minimizing geodesic of $X$ between two points of $Y$. In Definition 1.1, we also say $Y$ has arc/chord curvature $\leq A$. An equivalent condition is that for any $A^{\prime}>A$, there is $s\left(A^{\prime}\right)>0$ such that for every pair of points with distance $s<s\left(A^{\prime}\right)$ in $Y$, the chord joining them is longer than for a circular arc of length $s$ and curvature $A^{\prime}$ in the plane. This explains the choice of constant $\left(A^{2} / 24\right)$ in (1.1). (See Remark 3.2.)

If $X$ is Riemannian and $Y$ is any compact subset, then by a theorem of Lytchak, a condition equivalent to such a bound is positive reach, namely, uniform neighborhoods in which $Y$ has the unique footpoint property [L2]. If $Y$ is an embedded Riemannian submanifold, (1.1) is equivalent to a bound $|I I| \leq A$ on the second fundamental form.

In $\mathrm{CAT}(K)$ spaces, curves with extrinsic curvature $\leq A$ have the globalization property, namely, they satisfy sharp global arc/chord bounds [AB1].

Subsets of extrinsic curvature $\geq A$ are defined analogously (see Definition 3.1). In $\S 4$ and $\S 5$, we also hypothesize other versions of extrinsic curvature $\geq A$, obtained by using base-angle/chord comparisons rather than arc/chord comparisons.

1.2. Basics. A geodesic will always be a locally minimizing curve parametrized by arclength.

A locally Lipschitz function $f$ on $X$ is $\lambda$-concave for $\lambda \in \mathbf{R}$ if for any geodesic $\gamma$, the function

$$
f \circ \gamma(t)-\lambda t^{2} / 2
$$

is concave. The locally Lipschitz condition can be relaxed to continuity alone when $X$ is CBB [Pt2, 3.3.2]. We write $f^{\prime \prime}(p) \leq \lambda$ if for any $\epsilon>0$, there is a neighborhood $U$ of $p$ such that the restriction of $f$ to $U$ is $(\lambda+\epsilon)$-concave. By

$$
f^{\prime \prime} \leq g
$$

for a function $g$ on $X$, we mean $f^{\prime \prime}(p) \leq g(p)$ for all $p \in X$. A function $f$ is called semiconcave if (1.3) holds for some $g$. We call a $\lambda$-concave function very concave when $\lambda<0$, and almost concave when $\lambda>0$. Exactly analogous definitions hold with convex substituted for concave and inequalities reversed.

The differential inequalities considered in this paper are those most closely tied to the geometry of Alexandrov spaces, namely

$$
f^{\prime \prime}+K f \leq 1, f^{\prime \prime}+K f \geq 1,
$$

and

$$
f^{\prime \prime}+K f \leq 0, f^{\prime \prime}+K f \geq 0 .
$$


The inequalities (1.4) tend to be used when the motivating idea is distance from a point, and the inequalities (1.5), when it is distance from a geodesic. If $K \neq 0$, the solution sets of (1.4) and (1.5) differ by the constant $1 / K$.

The infinitesimal support properties that define (1.4) and (1.5) convert to the global support properties that we use in this paper. Namely, a solution's restriction to a geodesic of length $<\pi / \sqrt{K}$ is supported on the appropriate side by the solution of the corresponding differential equation, assuming either the same endpoint values or the same initial value and derivative. (Throughout, $\pi / \sqrt{k}=\infty$ if $k \leq 0$.)

Recall that $X$ has CBB by $K$ if and only if for every $q \in X$, the modified distance from $q, f=\operatorname{md}_{K} \circ \operatorname{dist}_{q}$, satisfies

$$
f^{\prime \prime}+K f \leq 1
$$

on the open ball $B(q, \pi / \sqrt{K})$, where

$$
\operatorname{md}_{K}(x)= \begin{cases}x^{2} / 2, & K=0 \\ (1 / K)(1-\cos \sqrt{K} x), & K>0 \\ (1 / K)(1-\cosh \sqrt{-K} x), & K<0 .\end{cases}
$$

For $\operatorname{CAT}(K)$, the defining inequality in (1.6) is reversed, namely, $f=\operatorname{md}_{K} \circ$ dist $_{q}$ satisfies

$$
f^{\prime \prime}+K f \geq 1
$$

on $B(q, \pi / \sqrt{K})$. For CBA by $K$, the condition is imposed locally.

The differential inequalities (1.6) and (1.8) on $f=\operatorname{md}_{K} \circ$ dist $_{q}$ are equivalent to the classical definitions of Alexandrov spaces in terms of distance comparisons between triangles in $X$ and their model triangles in the constantly curved model plane $S_{K}$. Indeed, the inequalities become equations in $S_{K}$. Since along geodesics, solutions of the differential inequality are supported by solutions of the equation, then distances in $X$ compare to distances in $S_{K}$.

Let

$$
\operatorname{sn}_{K}(x)=\operatorname{md}_{K}{ }^{\prime}(x)= \begin{cases}x, & K=0 \\ (1 / \sqrt{K}) \sin (\sqrt{K} x), & K>0 \\ (1 / \sqrt{-K}) \sinh (\sqrt{-K} x), & K<0 .\end{cases}
$$

For CBB spaces, Perelman's concavity theorem [Pr], with its formulation made more exact in [AB2], states that $f=\mathrm{sn}_{K} \circ \operatorname{dist}_{\partial X}$ satisfies

$$
f^{\prime \prime}+K f \leq 0,
$$

where dist $_{\partial X}$ is the distance from the boundary $\partial X$. The inequality becomes an equation when $X$ is a model halfplane.

1.3. Statement of Results. Now we describe our results more precisely:

1.3.1. Extrinsic and intrinsic curvature bounds on subspaces. In Section 2 we derive upper bounds on the extrinsic curvature of the superlevels of almost concave functions. By changing the sign, it is equivalent to consider sublevels of almost convex functions.

Lytchak [L3] proved the existence of some extrinsic curvature bound in this setting, and we build on his work. Our estimates in Theorems 1.3 and 1.6 are sharp in the sense that in the Riemannian setting they become $\lambda /\left|\nabla_{p} f\right|$, which is the correct formula. We do not know whether the bound in Theorem 1.3 can 
be improved to $\lambda /\left|\nabla_{p} f\right|$ or even more strongly to $\lambda / g$. However, the theorem immediately implies that the bound is at most $\sqrt{2} \lambda / g$.

Let us say a subset $Y$ of $X$ has extrinsic curvature $\leq A$ at $p \in Y$ if

$$
\limsup _{\epsilon \rightarrow 0} \sqrt{24(s-r) / r^{3}} \leq A
$$

where limsup is taken over all chords of the metric ball $B_{Y}(p, \epsilon)$ in $Y$. For extrinsic curvature $\geq A$ at $p$, substitute $\liminf$ for $\lim \sup$, and $\geq$ for $\leq$. (We use these notions of pointwise extrinsic curvature bound only to state Theorems 1.3 and 1.6. These theorems are immediate corollaries of slightly stronger uniform statements in Sections 2 and 3.)

Theorem 1.3. Let $X$ be a $C B A$ or $C B B$ space, and $f: \operatorname{Dom} f \rightarrow \mathbf{R}$ be a $\lambda$-concave function with $\lambda>0$, where $\operatorname{Dom} f$ is an open subset of $X$. Let $Y=f^{-1}([c, \infty))$ be a superlevel of $f$. If $g>0$, then the extrinsic curvature of $Y$ at $p \in Y$ is at most

$$
\lambda \sqrt{\frac{2}{g^{2}}-\frac{1}{G^{2}}}
$$

where $g$ and $G$ are the $\liminf$ and $\limsup$ respectively of $\left|\nabla_{q} f\right|$ as $q \rightarrow p$ within $f^{-1}((-\infty, c))$.

Remark 1.4. The bound in Theorem 1.3 is well-defined if $\left|\nabla_{p} f\right|>0$, by the semicontinuity of $\left|\nabla_{p} f\right|$ [Pt2, Cor. 1.3.5]. That is,

$$
\left|\nabla_{p} f\right|=\liminf _{q \rightarrow p}\left|\nabla_{q} f\right| \leq g \leq G \leq \limsup _{q \rightarrow p}\left|\nabla_{q} f\right| .
$$

The left equation holds because on a gradient curve $f$ is also $\lambda$-concave (same reference) with right-derivative of $f$ equal to $\left|\nabla_{p} f\right|$, so that the limit at $p$ through the superlevel is at most $\left|\nabla_{p} f\right|$. The interior inequalities hold because lim inf and limsup are monotone on subsets, in particular, on $f^{-1}((-\infty, c)) \subset X$.

The proof of Theorem 1.3, together with theorems on the Gauss Equation and the injectivity radii of subspaces proved in [AB4], implies the following new intrinsic curvature bounds:

Corollary 1.5. Let $X$ be a $C A T(K)$ space, and $f: X \rightarrow \mathbf{R}$ be a $\lambda$-concave function with $\lambda>0$. Let $Y=f^{-1}([c, \infty))$ be a superlevel of $f$, and $Z=f^{-1}(c)$ be the corresponding level. Suppose $G \geq|\nabla f| \geq g>0$ on a collar of $Z$ in the sublevel $f^{-1}((-\infty, c))$. Then $Y$ in its intrinsic metric has $C B A$ by

$$
K^{\prime}=K+\lambda^{2}\left(\frac{2}{g^{2}}-\frac{1}{G^{2}}\right),
$$

and the injectivity radius of $Y$ is at least $\pi / \sqrt{K^{\prime}}$.

In Section 3, we find lower bounds, analogous to the upper bounds in Theorem 1.3 , for the extrinsic curvature of level sets of very concave functions. Their superlevels and sublevels usually have nonempty interiors and so have no positive lower extrinsic curvature bound.

Theorem 1.6. Let $X$ be a $C B A$ or $C B B$ space, and $f: \operatorname{Dom} f \rightarrow \mathbf{R}$ be a $\lambda$-concave function with $\lambda<0$, where $\operatorname{Dom} f$ is an open subset of $X$. Let $Y=f^{-1}(c)$ be a level of $f$. If $0<G \leq \sqrt{2} g$, then the extrinsic curvature of $Y$ at $p \in Y$ is at least

$$
|\lambda| \sqrt{\frac{2}{G^{2}}-\frac{1}{g^{2}}}
$$


where $g$ and $G$ are the liminf and limsup respectively of $\left|\nabla_{q} f\right|$ as $q \rightarrow p$ within $f^{-1}((c, \infty))$.

1.3.2. CBB spaces with extrinsically curved boundary in the base-angle sense. Theorem 1.8, which is proved in Section 4, provides important examples of functions to which Theorem 1.6 applies. Moreover, because these functions reflect the global geometry of their underlying spaces, Theorem 1.8 has global structural consequences.

Definition 1.7. (Model region and model radius)

(1) Let $\widetilde{X}(K, A)$ be the convex domain in $S_{K}$ bounded by the curve of constant curvature $A \neq 0$ : a circle if $K>-A^{2}$, a curve equidistant from a geodesic if $K<-A^{2}$, and a horocycle if $K=-A^{2}$.

(2) Let $R(K, A)$ denote the radius of the boundary circle of $\widetilde{X}(K, A)$ if $K>$ $-A^{2}$, and the distance of the boundary equidistant curve to its core geodesic if $K<-A^{2}$. If $K=-A^{2}$, let $R(K, A)=\infty$.

A point $p \in \partial X$ is called a footpoint if $p$ is an endpoint of a minimizer from a point $q \in X-\partial X$ to $\partial X$. The minimizer from $q$ to $p$ is unique and orthogonal to the direction space $\Sigma_{p} \partial X$ of $\partial X$, and the direction space $\Sigma_{p} X$ is the spherical cone over $\Sigma_{p} \partial X[\mathrm{Pr}]$.

Theorem 1.8. Suppose $X$ is a finite-dimensional space of $C B B$ by $K$, and $\partial X \neq \emptyset$ has extrinsic curvature $\geq A>0$ in the base-angle sense at each footpoint $p \in \partial X$. If $K \neq-A^{2}$, set

$$
\mathcal{D}=R(K, A)-\operatorname{dist}_{\partial X} .
$$

(a) If $K>-A^{2}$, then $\mathcal{D}$ is nonnegative, and $f=\operatorname{md}_{K} \mathcal{D}$ satisfies $f^{\prime \prime}+K f \geq 1$.

(b) If $K=-A^{2}$, then $f=\exp \sqrt{-K}\left(-\operatorname{dist}_{\partial X}\right)$ satisfies $f^{\prime \prime}+K f \geq 0$.

(c) If $K<-A^{2}$, then $f=\sinh \sqrt{-K} \mathcal{D}$ satisfies $f^{\prime \prime}+K f \geq 0$.

These differential inequalities on $\operatorname{dist}_{\partial X}$ are strictly stronger than the corresponding inequalities (1.9) on the function $\operatorname{sn}_{K} \circ$ dist $\partial X$ in Perelman's concavity theorem, reflecting our curvature assumption on the boundary.

As an example of part (a), we may take $X$ to be a ball of radius $R(K, A)$ and center $\widetilde{q}$ in $S_{K}^{n}$. Then $\mathcal{D}=\operatorname{dist}_{\widetilde{q}}$, so the inequality on $f=\operatorname{md}_{K} \mathcal{D}$ becomes the equation governing the distance from $\widetilde{q}$ to any geodesic in $S_{K}^{n}$. By analogy, in part (a) we may regard $\mathcal{D}$ as "distance to a ghost center", and the inequality on $f=\mathrm{md}_{K} \mathcal{D}$ as a $\operatorname{CAT}(K)$-type condition, as in (1.8), pulling the interiors of geodesics towards the ghost center.

The condition $f^{\prime \prime}+K f \geq 1$ on $f=\operatorname{md}_{K} \mathcal{D}$ in part (a) is a unifying formulation, independent of the sign of $K$. For $0>K>-A^{2}$, it is trivially equivalent to $f=\cosh \sqrt{-K} \mathcal{D}$ satisfying $f^{\prime \prime}+K f \geq 0$, and hence is analogous to the conditions in parts (b) and (c).

The global nature of Theorem 1.8 yields the following structure theorems.

Corollary 1.9. Suppose $X$ is a finite-dimensional space of $C B B$ by $K$, and $\partial X \neq \emptyset$ has extrinsic curvature $\geq A>0$ in the base-angle sense at each footpoint $p \in \partial X$. If $K>-A^{2}$, then:

(1) The radius of $X$ is $\leq R(K, A)$.

(2) The length of any geodesic in $X$ is $\leq 2 R(K, A)$.

(3) dist $_{\partial X}$ is concave, and its maximum set consists of a single point. In particular, $X$ has a 1-Lipschitz strong deformation retraction to a point. 
For Riemannian manifolds with boundary, Corollary 1.9(1),(2) are due to Dekster [D] (see Remark 4.4).

Corollary 1.10 (Rigidity). Suppose $X$ is a finite-dimensional space of $C B B$ by $K$, and $\partial X \neq \emptyset$ has extrinsic curvature $\geq A>0$ in the base-angle sense at each footpoint $p \in \partial X$.

(1) If $K>-A^{2}$ and the radius of $X$ is $R(K, A)$, then $X$ is isometric to a ball of radius $R(K, A)$ about the vertex in a $K$-cone.

(2) If $K=-A^{2}$ and the radius of $X$ is $\infty$, then $X$ is isometric to a horoball in an ideal $K$-cone.

Finally, in Section 5 we examine the relation between base-angle/chord and arc/chord curvature bounds. In particular we show that if the boundary of a CBB space has base-angle/chord curvature $\geq A>0$, then it has arc/chord curvature $\geq A>0$.

1.4. Background. Analogues of the Gauss Equation, governing the passage from extrinsic curvature bounds to intrinsic ones, tend to be challenging in the Alexandrov setting. For instance, a major unsolved problem in the theory of CBB spaces is whether the boundary of a convex set inherits a curvature bound.

In CBA spaces, more is known. Ruled surfaces inherit a curvature bound above by a classical theorem of Alexandrov [A]. It is an open question whether saddle surfaces inherit such a bound, but Mese showed that minimal surface immersions do so $[\mathrm{M}]$, and Petrunin showed that 'metric minimizing' surfaces do so [Pt1]. For subsets with extrinsic curvature $\leq A$ in CBA spaces, the existence of some intrinsic curvature bound above was proved in [L1], and the Gauss Equation was proved in [AB4].

Semiconcave functions and their associated subspaces capture much of the geometry of Alexandrov spaces, both CBB and CBA. Gradient curves of semiconcave functions were introduced and studied in $[\mathrm{PP}]$ (for $\mathrm{CBB}$ spaces) and their properties are developed in [L3] (for both CBB and CBA) and [Pt2]. In particular, in our settings gradient curves exist and are unique, and the corresponding gradient flow is locally Lipschitz.

\section{Almost concave functions}

The pointwise estimate of Theorem 1.3 is an immediate consequence of the following theorem. Given $Z \subset Z^{\prime} \subset X$, by a $\delta$-collar of $Z$ in $Z^{\prime}$ we mean the subset of $Z^{\prime}$ at distance $<\delta$ from $Z$, where $\delta>0$.

Theorem 2.1. Let $X$ be a $C B A$ or $C B B$ space, and $f: \operatorname{Dom} f \rightarrow \mathbf{R}$ be a $\lambda$-concave function with $\lambda>0$, where $\operatorname{Dom} f$ is an open subset of $X$. Let $Y=f^{-1}([c, \infty))$ be a superlevel, and $Z$ be an open subset of the level $f^{-1}(c)$ with $\operatorname{cl}(Z) \subset \operatorname{Dom} f$. Suppose $G \geq|\nabla f| \geq g>0$ on a $\delta$-collar $\mathcal{C}$ of $Z$ in the sublevel $f^{-1}((-\infty, c))$. Then the extrinsic curvature of $Y$ on $Z$ is at most

$$
A=\lambda \sqrt{\frac{2}{g^{2}}-\frac{1}{G^{2}}}
$$

Philosophy of proof. We prove that for any chord $\sigma$ of $Z$ of length $r<r_{0}$, there is a curve $\tau$ in $Y$ between the ends of $\sigma$ satisfying

$$
s-r \leq\left(A^{2} / 24\right) r^{3}+C r^{4},
$$


where $r_{0}$ and $C$ depend only on $\lambda, g, G, \delta$. Throughout we use $O\left(r^{4}\right)$ notation to convey this uniformity, and similarly for $O\left(r^{k}\right)$. Note that our conclusion is stronger than is implied by Definition 1.1, not only because of the form of (2.1) but also because it holds for all $r<r_{0}$.

Lytchak obtains his extrinsic curvature bound in [L3] by estimating the growth of half of a chord as the midpoint is pushed along the gradient curve until it reaches $Y$. A natural refinement of this technique would be to partition the chord into small segments and estimate their growth as the ends are pushed along gradient curves. Although this refinement improves the extrinsic curvature estimate, that improvement is still not sharp. We find that if instead each small segment is pushed by moving the end with lower $f$-value until both ends are at the same level, and then by moving both ends along equal levels until they reach $Y$, the overestimate due to the second motion is exactly offset by the decrease during the first motion (see Figure 2). The estimate on the combined motions yields the theorem.

Proof. Take $c=0$, so that $Y=f^{-1}[0, \infty)$.

(a) Parametrizing $\sigma$ on $[-r / 2, r / 2]$, we may assume $f \circ \sigma \mid(-r / 2, r / 2)<0$. Indeed, suppose the theorem has been proved for that case, and consider a chord $\sigma$ of $Z$ for which the interior is not entirely outside $Y$. Decompose $\sigma$ into countably many open segments $\left\{\sigma_{i}\right\}$ of lengths $r_{i}$ on which $f<0$, with the remainder $\bar{\sigma}$ in $Y$; let $\tau_{i}$ be a suitably short curve in $Y$ of length $s_{i}$ connecting the ends of $\sigma_{i}$ for each $i$. Take $\tau$ to be the chain of $\bar{\sigma}$ with all the $\tau_{i}$. Since the length measure of $\bar{\sigma}$ contributes equally to the lengths $r$ and $s$ of $\sigma$ and $\tau$, the difference is entirely due to the differences $s_{i}-r_{i}$, and so:

$$
\begin{aligned}
s-r & =\sum_{i} s_{i}-r_{i} \\
& \leq \sum_{i}\left(\frac{A^{2}}{24} r_{i}^{3}+C r_{i}^{4}\right) \\
& \leq \frac{A^{2}}{24}\left(\sum_{i} r_{i}\right)^{3}+C\left(\sum_{i} r_{i}\right)^{4} \\
& \leq \frac{A^{2}}{24} r^{3}+C r^{4} .
\end{aligned}
$$

(b) Since $f \circ \sigma(x)-\lambda x^{2} / 2$ is concave on $[-r / 2, r / 2]$, with value $-\lambda r^{2} / 8$ at both endpoints,

$$
0 \geq f(\sigma(x))-\lambda x^{2} / 2 \geq-\lambda r^{2} / 8 .
$$

For each $x \in[-r / 2, r / 2]$, let $\eta_{x}$ be the gradient curve starting at $\sigma(x)$. See Figure 1. By assumption, there is a $\delta$-collar $\mathcal{C}$ of $Z$ in $f^{-1}((-\infty, c))$ such that $G \geq|\nabla f| \geq g>0$ on $\mathcal{C}$. Since $\operatorname{cl}(Z) \subset \operatorname{Dom} f$, we may choose $\delta$ so that $\mathcal{C} \subset \operatorname{Dom} f$. We wish only to consider chords $\sigma$ of $Z$ whose length $r$ is so small that $\eta_{x}$ remains in $\mathcal{C}$ until reaching a point of $f^{-1}(0)$, which we take to be the righthand endpoint of $\eta_{x}$. Since the distance from any point on $\sigma$ to $Z$ is at most $r / 2$, it suffices by (2.2) to take $r / 2+\lambda r^{2} / 8 g<\delta$, say

$$
r<r_{0} \stackrel{\text { def }}{=} \min \{\sqrt{8 g \delta /(\lambda+4 g)}, 1\} .
$$


It is convenient to reparametrize the gradient curves $\eta_{x}$ by the values of $f$. Then the righthand velocity vector is $\nabla f /|\nabla f|^{2}$ at each point. Set

$$
\tau(x)=\eta_{x}(0) \text {. }
$$

Since the gradient flow is locally Lipschitz, $\tau$ is rectifiable. Our goal is a sharp upper bound on $\frac{d u}{d x}$, where $u$ is the arclength parameter of $\tau$.

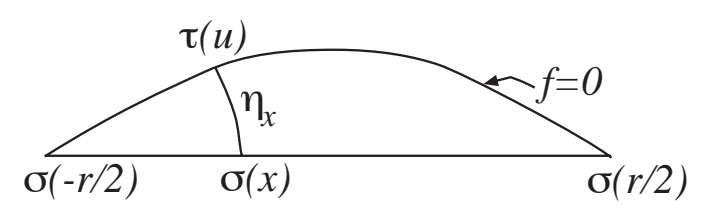

Figure 1. Chord $\sigma$ and gradient projection $\tau$

(c) As two points move up gradient curves $\eta_{x}$ and $\eta_{x+\Delta x}$, the rate of change of the distance between them is the sum of two terms, each of which has the form

$$
\text { (speed of end point) } \cdot \cos \theta \text {, }
$$

where $\theta$ is the angle between a minimizer connecting the two points and the path traveled by the end point. If there is more than one minimizer, the one used must be a limit of minimizers between the end points (see [BBI, p, 126]).

As noted above, we are going to break the variation into two stages, as illustrated by Figure 2. In the equilevel stage, the two ends move up the gradient curves at the same $f$-levels, until they reach $Y$. In the triangular stage, one end moves upward until it reaches the same level as the other end.

We begin by summarizing the basic estimates obtained in the equilevel stage, by integrating the first variation of distance between two points moving up gradient curves $\eta_{x}(y)$ and $\eta_{x+\Delta x}(y)$.

Let the minimizer connecting $p=\eta_{x}(y)$ and $q=\eta_{x+\Delta x}(y)$ be $\gamma_{y}$, and $L(y)=$ $d(p, q)$ be its length. Set $v_{p}=\gamma_{y}^{\prime}(0)$. Since $f$ is semiconcave,

$$
D_{p} f\left(v_{p}\right)=\left(f \circ \gamma_{y}\right)_{+}^{\prime}(0) \leq|\nabla f|(p) \cos \theta_{p}
$$

where $\theta_{p}$ is the angle between $w_{p}=\frac{\nabla f}{|\nabla f|}$ and $v_{p}$. This gives an inequality for the first variation term at the left endpoint of $\gamma_{y}$. The term at the right endpoint is calculated similarly, and combining them gives the following inequality for the first variation (recalling that the speed of $\eta_{x}$ is $1 /|\nabla f|(p)$ ):

$$
L_{+}^{\prime}(y) \leq \frac{\left(f \circ \gamma_{y}\right)_{-}^{\prime}(L(y))}{|\nabla f|^{2}(q)}-\frac{\left(f \circ \gamma_{y}\right)_{+}^{\prime}(0)}{|\nabla f|^{2}(p)}
$$

Since $f \circ \gamma_{y}(t)-t^{2} / 2$ is concave,

$$
\left(f \circ \gamma_{y}\right)_{-}^{\prime}(L) \leq \lambda L / 2, \quad-\left(f \circ \gamma_{y}\right)_{+}^{\prime}(0) \leq \lambda L / 2 .
$$

A common lower bound $|\nabla f|^{2} \geq g^{2}$ applies to the terms in (2.3), so

$$
L_{+}^{\prime}(y) \leq \frac{\lambda L(y)}{g^{2}} .
$$


Dividing by $L(y)$ and integrating on $c_{0} \leq y \leq 0$ gives:

$$
\log \frac{L(0)}{L\left(c_{0}\right)} \leq-\frac{\lambda c_{0}}{g^{2}}
$$

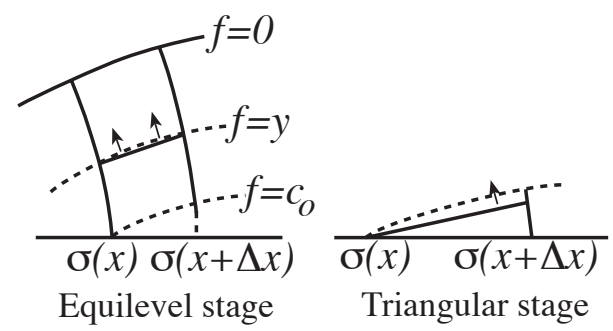

Figure 2. Variation from $\Delta x$ to $\Delta u$ in two stages

(d) Exponentiating and expanding in (2.5) will now give an upper bound for $L(0)-L\left(c_{0}\right)$, which we combine with the bound $(2.2)$ on $c_{0}$, namely,

$$
c_{0}=f(\sigma(x))=O\left(r^{2}\right) .
$$

The result is then

$$
L(0)-L\left(c_{0}\right) \leq-\frac{\lambda c_{0}}{g^{2}} L\left(c_{0}\right)+L\left(c_{0}\right) O\left(r^{4}\right) .
$$

(e) Now we deal with the remaining discrepancy between $L(0)$ and $\Delta x$, by applying first variation to a small "almost triangle". We may assume that initially $\sigma(x+\Delta x)$ is at a lower level than $\sigma(x)$, since otherwise we could reverse the direction of $\sigma$. Thus $f(\sigma(x))=c_{0} \leq y \leq 0$. We only need the contribution of this triangular stage when $(f \circ \sigma)_{+}^{\prime}(x)<0$. For if $(f \circ \sigma)_{+}^{\prime}(x)=0$, then the length of the initial segment of $\eta=\eta_{x+\Delta x}$ has an estimate $o(\Delta x)$, and hence the same estimate holds for the change given by integrating the first variation for that segment. Thus, the contribution of the first stage to $\frac{d u}{d x}$ will be 0 .

Assuming $(f \circ \sigma)_{+}^{\prime}(x)<0$ and

$$
c_{0}=f(\sigma(x))>c_{1}=f(\sigma(x+\Delta x)),
$$

let $\gamma_{y}$ be a minimizer from $\sigma(x)$ to $\eta(y), c_{0} \geq y \geq c_{1}$. Now the first variation for $L(y)=\left|\gamma_{y}\right|=\mid(\sigma(x) \eta(y) \mid$ has only one term,

$$
L_{+}^{\prime}(y) \leq \frac{\left(f \circ \gamma_{y}\right)_{-}^{\prime}(L(y))}{|\nabla f|^{2}(q)},
$$

where $q=\gamma_{y}(L(y))$

(f) In order to proceed we need a good upper bound on $c_{0}-c_{1}$, as defined in (2.8). By the concavity of $F(x)=f \circ \sigma(x)-\lambda x^{2} / 2$ on $[-r / 2, r / 2]$, we have $F^{\prime}(-r / 2) \leq \lambda r / 2$ and $F^{\prime}(r / 2) \geq-\lambda r / 2$. Since $F^{\prime}$ is nonincreasing,

$$
-\lambda r \leq F^{\prime}(r / 2)+\lambda x \leq(f \circ \sigma)^{\prime}(x) \leq F^{\prime}(-r / 2)+\lambda x \leq \lambda r .
$$

Immediately it follows that

$$
c_{0}-c_{1} \leq \lambda r \Delta x
$$


(g) We also need a better estimate for $L(y)$ than that given by the Lipschitz continuity of the gradient flow. For our immediate goal a triangle inequality will suffice: the length of an arc of a gradient curve $\eta$ is bounded by its speed, at most $1 / G$, times the parameter change, $c_{0}-y$, so $\left|\eta\left(\left[y, c_{0}\right]\right)\right|=\Delta x O(r)$. Chaining the segment of $\sigma$ of length $\Delta x$ with $\eta$ then gives

$$
L(y)=\Delta x(1+O(r)), c_{1} \leq y \leq c_{0} .
$$

(h) The concavity of $f \circ \gamma_{y}(t)-\lambda t^{2}$ implies

$$
\left(f \circ \gamma_{y}\right)_{-}^{\prime}(L)-\lambda L \leq \frac{y-\lambda L^{2} / 2-c_{0}}{L} .
$$

Hence from (2.9) and (2.11),

$$
L_{+}^{\prime}(y) \leq\left(-\frac{c_{0}-y}{G^{2} \Delta x}+\frac{\lambda \Delta x}{2 g^{2}}\right)(1+O(r)) .
$$

Integrating on $c_{1} \leq y \leq c_{0}$,

$$
L\left(c_{0}\right)-\Delta x \leq\left(-\frac{\left(c_{0}-c_{1}\right)^{2}}{2 G^{2} \Delta x}+\frac{\lambda^{2}(\Delta x)^{2} r}{2 g^{2}}\right)(1+O(r)) .
$$

(i) Now add the inequalities (2.7) and (2.12), again applying (2.11), to obtain a bound on $L(0)$ due to both stages:

$$
L(0) \leq \Delta x+\left(\frac{-\lambda c_{0} \Delta x}{g^{2}}+\left[\frac{-\left(c_{0}-c_{1}\right)^{2}}{2 G^{2} \Delta x}+\frac{\lambda^{2}(\Delta x)^{2} r}{2 g^{2}}\right][1+O(r)]\right)+\Delta x O\left(r^{3}\right) .
$$

Divide by $\Delta x$, let $\Delta x \rightarrow 0$, and change back to the notation $c_{0}=f(\sigma(x)), c_{1}=$ $f(\sigma(x+\Delta x)$ :

$$
\frac{d u}{d x} \leq 1-\lambda\left(\frac{f \circ \sigma(x)}{g^{2}}+\frac{\left((f \circ \sigma)_{+}^{\prime}(x)\right)^{2}}{2 G^{2} \lambda}\right)+O\left(r^{3}\right) .
$$

The error terms have been combined, using $(f \circ \sigma)_{+}^{\prime}(x)=O(r)$ and $f(\sigma(x))=O\left(r^{2}\right)$ (by (2.6)). Integrate this inequality on $-r / 2 \leq x \leq r / 2$ to obtain a bound on the length of $\tau$ :

$$
|\tau| \leq r-\lambda \int_{-r / 2}^{r / 2}\left(\frac{z}{g^{2}}+\frac{\left(z^{\prime}\right)^{2}}{2 G^{2} \lambda}\right) d x+O\left(r^{4}\right),
$$

where $z=f(\sigma(x))$. Here the Riemann integral exists because the integrand is bounded and its set of discontinuities has measure zero. We claim

$$
I \stackrel{\text { def }}{=}-\lambda \int_{-r / 2}^{r / 2}\left(\frac{z}{g^{2}}+\frac{\left(z^{\prime}\right)^{2}}{2 G^{2} \lambda}\right) d x \leq \frac{\lambda^{2} r^{3}}{24}\left(\frac{2}{g^{2}}-\frac{1}{G^{2}}\right) .
$$

(j) Let

$$
w=z+\frac{\lambda}{8}\left(r^{2}-4 x^{2}\right) .
$$

Both $z$ and $w$ vanish at the endpoints of $[-r / 2, r / 2]$, and $w$ is concave since $z$ is $\lambda$-concave. Set

$$
J=\int_{-r / 2}^{r / 2}\left(\frac{-\lambda w}{g^{2}}-\frac{\left(w^{\prime}\right)^{2}+2 \lambda x w^{\prime}}{2 G^{2}}\right) d x
$$

Then

$$
I=J+\frac{\lambda^{2} r^{3}}{24}\left(\frac{2}{g^{2}}-\frac{1}{G^{2}}\right) .
$$


Now we show $J<0$. To see this, regard (2.15) as defining a functional $J(w)$, and for fixed $w$ consider the function $J(t) \stackrel{\text { def }}{=} J(t w)$. Then

$$
J(t)=A t^{2}+B t,
$$

where

$$
A=-\int_{-r / 2}^{r / 2} \frac{\left(w^{\prime}\right)^{2}}{2 G^{2}} d x, \quad B=-\lambda \int_{-r / 2}^{r / 2}\left(\frac{w}{g^{2}}+\frac{x w^{\prime}}{G^{2}}\right) d x,
$$

and ' denotes righthand derivative. But $\int_{-r / 2}^{r / 2}\left(x w^{\prime}+w\right) d x=0$. Indeed, since $x, w$ are continuous and $w^{\prime}$ exists everywhere, then $(x w)^{\prime}=x w^{\prime}+w$ everywhere. On $[-r / 2, r / 2]$,

$$
(x w)^{\prime}=\frac{d F}{d x} \text { a.e., } \quad F(x) \stackrel{\text { def }}{=} \int_{-r / 2}^{x}\left(x w^{\prime}+w\right) d x .
$$

Since $x w$ and $F$ are absolutely continuous, and both vanish at $x=-r / 2$, they are equal. Therefore

$$
B=-\lambda \int_{-r / 2}^{r / 2}\left(\frac{1}{g^{2}}-\frac{1}{G^{2}}\right) w d x .
$$

Since $\lambda>0, G>g>0$, and $w>0$ on $[-r / 2, r / 2]$, then $B<0$.

Since $A<0$, the graph of $J(t)$ is a downward parabola. Since $J$ vanishes at $t=0$ and $t=-B / A<0$, then $J=J(1)<0$. By (2.16), the claim (2.14) follows. By (2.13), the proof is complete.

Similar theorems may be obtained for sublevels $f^{-1}((-\infty, c])$ by applying Theorem 2.1 to $-f$; and for functions satisfying differential inequalities such as (1.4) or (1.5) by using the relation to $\lambda$-concavity. For example:

Corollary 2.2. Let $X$ be a $C B A$ or $C B B$ space, and $f: X \rightarrow \mathbf{R}$ be a locally Lipschitz function satisfying $f^{\prime \prime}+f \geq 0$. Let $Y=f^{-1}((-\infty, c])$ be a sublevel with $c>0$, and $Z$ be an open subset of the level $f^{-1}(c)$. Suppose $G \geq|\nabla(-f)| \geq g>0$ on a $\delta$-collar of $Z$ in the superlevel $f^{-1}((c, \infty))$. Then the extrinsic curvature of $Y$ on $Z$ is at most

$$
A=c \sqrt{\frac{2}{g^{2}}-\frac{1}{G^{2}}} .
$$

For CBA spaces, Theorem 2.1 leads to intrinsic curvature and injectivity radius bounds for subspaces, as described in Corollary 1.5. We will prove Corollary 1.5 by combining Theorem 2.1 with the following:

Theorem 2.3 ([AB4]). Suppose $N$ is a subspace of extrinsic curvature $\leq A$ in a $C A T(K)$ space. Then

(1) (Gauss Equation) $N$ is an Alexandrov space of curvature bounded above by $K+A^{2}$.

(2) The injectivity radius $\operatorname{inj}_{N}$ of $N$ satisfies

$$
\operatorname{inj}_{N} \geq \pi / \sqrt{K+A^{2}} .
$$

Remark 2.4. By considering a sphere of extrinsic curvature $A$ in $S_{K}$, it becomes clear that

$$
\pi / \sqrt{K+A^{2}}=c(A, K) / 2,
$$

where $c(A, K)$ is the circumference of a circle of curvature $A$ in $S_{K}$. Thus the injectivity radius given here is the same as in [AB4]. 
Proof of Corollary 1.5. By Theorem 2.1, $Y=f^{-1}([c, \infty))$ has extrinsic curvature $\leq A=\lambda \sqrt{2 / g^{2}-1 / G^{2}}$. By Theorem 2.3(1), $Y$ in its intrinsic metric has CBA by $K^{\prime}=K+A^{2}$. By Theorem 2.3(2), the injectivity radius of $Y$ is at least $\pi / \sqrt{K^{\prime}}$.

\section{VERY CONCAVE FUNCTIONS}

Now we consider $\lambda$-concave functions with $\lambda<0$.

Definition 3.1. A subset $Y$ of $X$ has extrinsic curvature $\geq A$ (or arc/chord curvature $\geq A$ ) if there is a function $F(s) \geq 0$ satisfying $\lim _{s \rightarrow 0}\left(F(s) / s^{3}\right)=0$, such that for every pair of points with distance $s<s_{0}$ in $Y$ and distance $r$ in $X$,

$$
s-r \geq\left(A^{2} / 24\right) s^{3}-F(s) .
$$

If we only consider pairs of points in an open subset $Z \subset Y$, we say $Y$ has extrinsic curvature $\geq A$ on $Z$.

Remark 3.2. For a curve of constant curvature $A$ in any model space, independent of the curvature of that model space,

$$
s-r=\left(A^{2} / 24\right) s^{3}+O\left(s^{5}\right) .
$$

Thus an equivalent form of Definition 3.1 is: For any $A^{\prime}<A$, there is $s\left(A^{\prime}\right)$ such that for every pair of points with distance $s<s\left(A^{\prime}\right)$ in $Y$, the chord joining them is shorter than for a circular arc of length $s$ and curvature $A^{\prime}$ in the plane.

Remark 3.3. On the right side of (3.1), $s$ could be replaced by $r$ since $s \geq r$. Similarly on the right side of (1.1) in Definition 1.1, $r$ could be replaced by $s$.

In Definition 3.1, if $F(s)=-C s^{4}$ for some $C>0$, and there is $s_{0}>0$ such that (3.1) holds for all $s<s_{0}$, then we can take $C_{1}=\max \left\{C, A^{2} / 24 s_{0}\right\}$ and show that $s-r \geq\left(A^{2} / 24\right) s^{3}-C_{1} s^{4}$ not only for $s<s_{0}$, but also for $s \geq s_{0}$; that is, Definition 3.1 is satisfied without the restriction $s<s_{0}$. It is this stronger form that we prove in Theorem 3.4.

The next theorem states that the levels of very concave functions have large extrinsic curvature. Theorem 1.6 is an immediate consequence.

Theorem 3.4. Let $X$ be a $C B A$ or $C B B$ space, and $f: \operatorname{Dom} f \rightarrow \mathbf{R}$ be a $\lambda$ concave function with $\lambda<0$, where $\operatorname{Dom} f$ is an open subset of $X$. Let $Y=f^{-1}(c)$ be a level of $f$, and $Z$ be an open subset of $Y$ with $\operatorname{cl}(Z) \subset \operatorname{Dom} f$. Suppose that $\sqrt{2} g \geq G \geq|\nabla f| \geq g>0$ on a $\delta$-collar $\mathcal{C}$ of $Z$ in the superlevel $f^{-1}((c, \infty))$. Then the extrinsic curvature of $Y$ on $Z$ is at least

$$
A=|\lambda| \sqrt{\frac{2}{G^{2}}-\frac{1}{g^{2}}} .
$$

Philosophy of proof. The proof is analogous to that of Theorem 2.1. However, there the proof starts with a geodesic in $X$ whose endpoints are on a superlevel $Y$, and follows gradient curves until they reach $Y$. Here the proof starts with a curve (an approximate geodesic) in a level $Y$ and follows gradient curves to some curve in $\mathcal{C}$. On each gradient curve we have a choice of when to stop, and we make a fortunate guess based on the model space pattern. 
Proof. Again we may assume $c=0$. Let $\tau$ be a curve of length $\bar{s}$ in $Y$ with ends $p, q \in Z$ and arclength parameter $u \in[-\bar{s} / 2, \bar{s} / 2]$. Define a curve $\sigma$ with parameter $u$ and the same ends as $\tau$, in terms of the gradient curves $\eta_{u}$ of $f$ starting at $\tau(u)$ :

$$
\sigma(u)=\eta_{u}\left(-\lambda\left[\bar{s}^{2}-4 u^{2}\right] / 8\right) .
$$

The curves $\eta_{u}$ are assumed to be parametrized by the values of $f$, so $f \circ \sigma(u)=$ $-\lambda\left[\bar{s}^{2}-4 u^{2}\right] / 8$. As in the proof of Theorem 2.1, for sufficiently small $\bar{s}$ relative to the collar width, due to the bounds on $|\nabla f|, \sigma$ will be in $\mathcal{C}$. Let $\bar{r}$ be the length of $\sigma$. Our immediate goal is to prove that for $\bar{s}<s_{0}$,

$$
\bar{s}-\bar{r} \geq \frac{A^{2}}{24} \bar{s}^{3}-C \bar{s}^{4},
$$

where $s_{0}$ and $C>0$ depend only on $\lambda, G, g, \delta$. Finally we shall let $s, r$ be distances between $p, q$ in $Y, X$, respectively, and taking $|\tau|$ to be arbitrarily close to $s$ will complete the proof.

For points $\tau(u), \tau(u+\Delta u)$ we consider the change in length from $|\tau(u) \tau(u+\Delta u)|$ to $|\sigma(u) \sigma(u+\Delta u)|$ in two stages: first, move both ends along equal levels until one end reaches $\sigma$, and secondly, move the other end until it reaches $\sigma$. For both stages, let $L(y)$ be the distance between the ends, where $y$ is the value of $f$ at the moving end or ends, and let $\gamma_{y}$ be a minimizer between those ends.

For the first (equilevel) stage, the inequality (2.3), derived from first variation, holds with no change. The next step estimating the values $\left(f \circ \gamma_{y}\right)^{\prime}$, is also the same, except that $G$ replaces $g$ in the inequality (2.4) due to the change in sign of $\lambda$ :

$$
L_{+}^{\prime}(y) \leq \frac{\lambda L}{G^{2}}
$$

Now assume that $u<0$, so it is the left end of $\gamma_{y}$ that reaches $\sigma$ first. The estimates we get for the length of $\sigma$ are then for the left half, but the same estimates hold for the other half by symmetry. Accordingly, let

$$
c_{0}=-\lambda\left(\bar{s}^{2}-4 u^{2}\right) / 8, \quad c_{1}=-\lambda\left[\bar{s}^{2}-4(u+\Delta u)^{2}\right] / 8 .
$$

As before, we integrate

$$
\frac{L^{\prime}}{L} \leq \frac{\lambda}{G^{2}}
$$

from $y=0$ to $y=c_{0}$ and exponentiate, obtaining the inequality corresponding to (2.7), but with the specific value of $c_{0}$ inserted:

$$
L\left(c_{0}\right) \leq L(0)\left(1-\frac{\lambda^{2}\left(\bar{s}^{2}-4 u^{2}\right)}{8 G^{2}}+O\left(\bar{s}^{4}\right)\right) .
$$

Since $L(0)$ is the chord length for an arc of $\tau$ of length $\Delta u$,

$$
L\left(c_{0}\right) \leq\left(1-\frac{\lambda^{2}\left(\bar{s}^{2}-4 u^{2}\right)}{8 G^{2}}+O\left(\bar{s}^{4}\right)\right) \Delta u
$$

Now we consider the remaining variation on the "triangular" piece, with $c_{0} \leq$ $y \leq c_{1}$. Inequality $(2.9)$ is exactly the same, $L_{+}^{\prime}(y) \leq\left(f \circ \gamma_{y}\right)_{-}^{\prime} L(y) /|\nabla f|^{2}(q)$.

Also the triangle inequality can be used in the same way to get the estimate for $L(y)$, but now we need a lower bound involving $L\left(c_{1}\right)$ and we have the specific value $c_{1}-c_{0}=\lambda(u+\Delta u / 2) \Delta u<\lambda u \Delta u$ :

$$
L(y) \geq L\left(c_{1}\right)-\lambda u \Delta u / g .
$$


As in Theorem 2.1(h), we get the same bound on $\left(f \circ \gamma_{y}\right)_{-}^{\prime}(L)$. In the next step, $G$ and $g$ are interchanged due to sign changes in $y-c_{0}$ and $\lambda$ :

$$
L_{+}^{\prime}(y) \leq \frac{y-c_{0}}{g^{2} L(y)}+\frac{\lambda L(y)}{2 G^{2}} \leq \frac{y-c_{0}}{g^{2} L(y)} \leq \frac{y-c_{0}}{g^{2}\left(L\left(c_{1}\right)-\lambda u \Delta u / g\right)} .
$$

There is a concern that if (3.5) is used immediately, then a small value of $L\left(c_{1}\right)$ in the denominator could make the resulting bound on $L_{+}^{\prime}(y)$ so large that it would be useless. But our goal is to show that $L\left(c_{1}\right)$ is small enough, so by breaking into two cases: $L\left(c_{1}\right) \geq(1-\bar{s}) \Delta u$, for which (3.5) can be used, and $L\left(c_{1}\right)<(1-\bar{s}) \Delta u$, for which a sufficiently small $\bar{s}$ is already enough control, a satisfactory inequality for both cases is obtained.

So first assume $L\left(c_{1}\right) \geq(1-\bar{s}) \Delta u$. Then $(3.5)$, provided that $-\bar{s}-\lambda u / g=O(\bar{s})$ is small, can be integrated on $c_{0} \leq y \leq c_{1}$ to obtain

$$
L\left(c_{1}\right) \leq L\left(c_{0}\right)+\frac{\lambda^{2} u^{2} \Delta u}{2 g^{2}}(1+O(\bar{s}))
$$

We add (3.4) and (3.6) to obtain

$$
L\left(c_{1}\right) \leq\left[1-\frac{\lambda^{2}\left(\bar{s}^{2}-4 u^{2}\right)}{8 G^{2}}+\frac{\lambda^{2} u^{2}}{2 g^{2}}(1+O(\bar{s}))\right] \Delta u,
$$

where the $O\left(\bar{s}^{4}\right)$ term has been subsumed by the term containing $u^{2} O(\bar{s})$.

Now for the case $L\left(c_{1}\right)<(1-\bar{s}) \Delta u$, if we take $\bar{s}$ so small that $\lambda^{2} \bar{s}^{2} / 8 G^{2}<\bar{s}$ and $\bar{s}<g /(2 g-\lambda)$, then (3.7) will also hold for those values of $u$ and $\Delta u$.

The left side of (3.7) is the chord length of the arc of $\sigma$ that corresponds to an arc of $\tau$ of length $\Delta u$. Since the right side is an even function of $u$, the inequality will also hold for $u>0$. If we take an arbitrary partition of $[-\bar{s} / 2, \bar{s} / 2]$ including the point 0 and add these inequalities with $u$ 's as partition points and $\Delta u$ 's as partition segment lengths, then the sum on the left becomes the polygonal approximation to the length $\bar{r}$ of $\sigma$, while the sum on the right becomes a Riemann sum for the integral

$$
\int_{-\bar{s} / 2}^{\bar{s} / 2}\left[1-\frac{\lambda^{2}\left(\bar{s}^{2}-4 u^{2}\right)}{8 G^{2}}+\frac{\lambda^{2} u^{2}}{2 g^{2}}(1+O(\bar{s}))\right] d u=\bar{s}-\frac{\lambda^{2}}{24}\left(\frac{2}{G^{2}}-\frac{1}{g^{2}}\right) \bar{s}^{3}+O\left(\bar{s}^{4}\right) .
$$

Now let $s, r$ be distances in $Z, \mathcal{C}$ and for any $\epsilon>0$ choose $\tau$ connecting the points in $Z$ with length $\bar{s}<s+\epsilon$. Then we construct $\sigma$ with length $\bar{r}$ corresponding to $\tau$, for which we have

$$
\bar{s}-\bar{r} \geq \frac{A^{2}}{24} \bar{s}^{3}-O\left(\bar{s}^{4}\right)
$$

Then since $\bar{r} \geq r$

$$
s+\epsilon-r \geq \frac{A^{2}}{24} s^{3}-O\left([s+\epsilon]^{4}\right),
$$

and taking $\epsilon \rightarrow 0$ obtains (3.1).

As before, there is a corollary corresponding to Corollary 2.2, giving a lower bound $-c \sqrt{\frac{2}{G^{2}}-\frac{1}{g^{2}}}$ for the extrinsic curvature of the levels $f^{-1}(c), c<0$, of functions satisfying $f^{\prime \prime}+f \geq 0$. 


\section{Curved Boundaries}

In this section, we take $X$ to be a finite-dimensional space of CBB by $K$, and prove Theorem 1.8.

Definition 4.1. The base angle at $p$ of a chord $\gamma$ of $\partial X$ at an endpoint $p$ is the angle formed by the direction of $\gamma$ at $p$ and $\Sigma_{p} \partial X$. The boundary $\partial X$ has extrinsic curvature $\geq A>0$ in the base-angle sense at $p$ if the base angles $\alpha$ at $p$ of chords of length $r$ from $p$ satisfy

$$
\liminf _{r \rightarrow 0} 2 \alpha / r \geq A \text {. }
$$

Remark 4.2. The base angle $\widetilde{\alpha}(r)$ of a chord of length $r$ for a curve of constant curvature $A$ in any model space, independent of the curvature of that model space, satisfies

$$
\widetilde{\alpha}(r)=A r / 2+O\left(r^{3}\right)
$$

(for further discussion, see $[\mathrm{AB} 1]$ ). Thus the following condition is equivalent to (4.1): the base angles of chords from $p$ satisfy

$$
\alpha \geq \widetilde{\alpha}(r)+o(r) .
$$

Recall that for given $K$ and $A$, Definition 1.7 specifies a model region

$$
\widetilde{X}=\tilde{X}(K, A)
$$

in $S_{K}$ and a model radius $R(K, A)$. Theorem 1.8 states that under an extrinsic curvature assumption on $\partial X$, the distance from $\partial X$ satisfies certain differential inequalities. We begin by verifiying that these inequalities become equations when $X$ is a model region.

Lemma 4.3. The differential inequalities that govern dist $_{\partial X}$ in Theorem 1.8 become equations when $X=\widetilde{X}$.

Proof. In part (a) of the theorem, $K>-A^{2}$ and $\widetilde{X}$ is a disk, say with center $\widetilde{q}$. Let

$$
\widetilde{\mathcal{D}}=R(K, A)-\operatorname{dist}_{\partial \widetilde{X}}=\operatorname{dist}_{\widetilde{q}},
$$

and $f=\operatorname{md}_{K} \circ \widetilde{\mathcal{D}}$. We verify that $f^{\prime \prime}+K f=1$.

The simplest case is exceptional, $K=0$, for which $f=\operatorname{dist}_{p}^{2} / 2$. It is trivially verified that $f^{\prime \prime}=1$.

Let $\operatorname{cs}_{K}$ be the real-valued function that satisfies $\operatorname{cs}_{K}^{\prime \prime}+K \operatorname{cs}_{K}=0, \operatorname{cs}_{K}(0)=$ $1, \operatorname{cs}_{K}^{\prime}(0)=0$. For $K \neq 0$ the constant term in $\operatorname{md}_{K}$ gives a constant term 1 in $K f$, so the claim that $\operatorname{md}_{K} \circ \widetilde{\mathcal{D}}$ satisfies $f^{\prime \prime}+K f=1$ reduces to the claim that $\operatorname{cs}_{K} \circ \operatorname{dist}_{\widetilde{p}}$ satisfies $f^{\prime \prime}+K f=0$, that is, $\operatorname{cs}_{K} \circ \operatorname{dist}_{\widetilde{p}}$ is $K$-affine. This fact follow from the general theory of $K$-affine functions on constant curvature model spaces of any dimension, as discussed in [AB2]; in the quadric hypersurface model they are the restrictions of linear functions on the ambient Euclidean (for $K>0$ ) or Minkowski (for $K<0$ ) space.

The differential equations used in parts (b) and (c) are established in the same way.

Remark 4.4. The above differential equations for $X=\widetilde{X}$ may be rewritten in classical form as 


$$
\widetilde{h}^{\prime \prime}=-\left(1-\left(\widetilde{h}^{\prime}\right)^{2}\right) A_{\widetilde{h}}(K, A),
$$

where $\widetilde{h}$ is the restriction of $\operatorname{dist}_{\partial \widetilde{X}}$ to a geodesic, and $A_{\mu}(K, A)$ is the curvature of the curve in $\widetilde{X}$ that is equidistant from $\partial \widetilde{X}$ at distance $\mu$. Specifically,

$$
A_{\mu}(K, A)= \begin{cases}\sqrt{K} \cot \sqrt{K}(R(K, A)-\mu), & K>0 \\ 1 /(R(K, A)-\mu), & K=0 \\ \sqrt{-K} \operatorname{coth} \sqrt{-K}(R(K, A)-\mu), & 0>K>-A^{2} \\ \sqrt{-K}, & K=-A^{2} \\ \sqrt{-K} \tanh \sqrt{-K}(R(K, A)-\mu), & K<-A^{2} .\end{cases}
$$

In [D], Dekster used these formulas and comparison arguments to obtain bounds on exit distances for geodesics and, more generally, curves of bounded curvature, in Riemannian domains of curvature $\geq K$ whose boundaries have a curvature bound $A$ with $K>-A^{2}$.

Now we are ready to prove Theorem 1.8. The hypothesis is that $\partial X$ has extrinsic curvature $\geq A>0$ in the base-angle sense at each footpoint.

Proof of Theorem 1.8. Since our goal is differential inequalities on - dist ${ }_{\partial X}$ that become equations in the models $\widetilde{X}$, it suffices to prove that the restriction of $\operatorname{dist}_{\partial X}$ to any given geodesic at any point is supported with subquadratic error by the restriction of $\operatorname{dist}_{\partial \widetilde{X}}$ to a geodesic. The negative sign then converts concavity inequalities to convexity ones.

Let $\tau:(-\epsilon, \epsilon) \rightarrow X$ be a geodesic. Since the claim is trivial if $\tau$ lies along a minimizer to the boundary, we may suppose there is no $\epsilon>0$ for which this is true. Set $q_{0}=\tau(0)$ and

$$
h=\operatorname{dist}_{\partial X} \circ \tau .
$$

Let $\gamma$ be a minimizer from $q_{0}$ to $\partial X$, say with footpoint $p_{0} \in \partial X$. The existence of such a minimizer follows from Perelman's doubling theorem, since there is a minimizer from $q_{0}$ to its copy in the double. We choose $\gamma$ to be the limit of minimizers from points $q_{i}=\tau\left(t_{i}\right), t_{i}>0$. Then $d h / d t\left(0^{+}\right)=-\cos \theta_{0}$, where $\theta_{0}$ is the minimum angle between $\tau \mid[0, \epsilon)$ and minimizers from $q_{0}$ to $\partial X$, and also the angle between $\tau \mid[0, \epsilon)$ and $\gamma$ ([BBI, p. 123-126]). In particular, $(d h / d t)\left(0^{-}\right)+$ $(d h / d t)\left(0^{+}\right) \leq 0$, that is, $h$ is infinitesimally concave.

Special case. If $K \leq-A^{2}$ we impose no restrictions, but if $K>-A^{2}$ we suppose

$$
\operatorname{dist}_{\partial X}\left(q_{0}\right)<R(K, A) .
$$

In $\widetilde{X}$, consider the model triangle $\widetilde{p}_{0} \widetilde{q}_{0} \widetilde{q}_{i}$. Then $\angle \widetilde{p}_{0} \widetilde{q}_{0} \widetilde{q}_{i} \uparrow \theta_{0}$. Let $\widetilde{z}_{i}$ be the footpoint of $\widetilde{q}_{i}$ on $\partial \widetilde{X}$. Let $\widetilde{\tau}$ be a maximal geodesic in $\widetilde{X}$ with $\widetilde{\tau}(0)=\widetilde{q}_{0}$ and making angle $\theta_{0}$ with the segment $\widetilde{q}_{0} \widetilde{p}_{0}$, and set

$$
\widetilde{h}=\operatorname{dist}_{\partial \widetilde{X}} \circ \widetilde{\tau} \text {. }
$$

Since $\angle \widetilde{p}_{0} \widetilde{q}_{0} \widetilde{q}_{i} \leq \theta_{0}$,

$$
\left|\widetilde{q}_{i} \widetilde{z}_{i}\right| \leq \widetilde{h}\left(t_{i}\right)
$$


Indeed, if $\theta_{0} \in\{0, \pi\}$, equality holds trivially. If $\theta_{0} \notin\{0, \pi\}$, opening the hinge $\widetilde{p}_{0} \widetilde{q}_{0} \widetilde{q}_{i}$, leaving $\widetilde{p}_{0} \widetilde{q}_{0}$ fixed, moves $\widetilde{q}_{i}$ away from $\partial \widetilde{X}$ transversely to the equidistant curve of $\partial \widetilde{X}$ if $\operatorname{dist}_{\partial \widetilde{X}}\left(q_{0}\right)<R(K, A)$, and moves $\widetilde{q}_{i}$ along the equidistant curve if $\operatorname{dist}_{\partial \widetilde{X}}\left(q_{0}\right)=R(K, A)$.

Let $\widetilde{\phi}_{i}$ be the angle at $\widetilde{p_{0}}$ between the chord $\widetilde{p_{0}} \widetilde{z_{i}}$ and $\partial \widetilde{X}$.

Write $q_{i}^{\prime}$ for the direction of a minimizer $p_{0} q_{i}$, and let $\xi_{i}$ be its projection to $\Sigma_{p_{0}} \partial X$. Passing to a convergent subsequence, let $\xi_{i} \rightarrow \xi \in \Sigma_{p_{0}} \partial X$. Setting $r_{i}=$ $\left|\widetilde{p_{0}} \widetilde{z_{i}}\right|$, we may choose points $p_{i} \in \partial X$ such that $d\left(p_{0}, p_{i}\right)=r_{i}$, and (writing $p_{i}^{\prime}$ for the direction of a minimizer $p_{0} p_{i}$ ) such that $p_{i}^{\prime}$ converges to $\lim \xi_{i}=\xi$. Let $\eta_{i}$ be the projection of $p_{i}^{\prime}$ to $\Sigma_{p_{0}} \partial X$, so $\eta_{i} \rightarrow \xi$.

Since the angle $\phi_{i}$ between $\eta_{i}$ and $p_{i}^{\prime}$ is no less than the base angle of the chord $p_{0} p_{i}$ of $\partial X$, then by hypothesis,

$$
\phi_{i} \geq \widetilde{\phi}_{i}+o\left(r_{i}\right)=\widetilde{\phi}_{i}+o\left(t_{i}\right) .
$$

Now set $\alpha_{i}=\angle q_{0} p_{0} q_{i}$ and $\beta_{i}=\angle \widetilde{q}_{0} \widetilde{p}_{0} \widetilde{q}_{i}$, so $\alpha_{i} \geq \beta_{i}$. Since $\Sigma_{p_{0}} X$ is the spherical cone over $\Sigma_{p_{0}} \partial X$, then in $\Sigma_{p_{0}} X$ the points $q_{0}^{\prime}, \xi_{i}, \eta_{i}$ are the vertices of an isometric copy of a standard spherical triangle which has left and right sides $q_{0}^{\prime} \xi_{i}$ and $q_{0}^{\prime} \eta_{i}$ respectively of length $\pi / 2$, and the base side $\xi_{i} \eta_{i}$ of length $\delta_{i}:=\left|\xi_{i} \eta_{i}\right| \rightarrow 0$. The points $q_{i}^{\prime}, p_{i}^{\prime}$ lie on the left and right sides respectively, where $\left|q_{0}^{\prime} q_{i}^{\prime}\right|=\alpha_{i}$ and $\left|p_{i}^{\prime} \eta_{i}\right|=\phi_{i} \rightarrow 0$. If we move from $q_{i}^{\prime}$ on the left side, first along a circular arc of radius $\alpha_{i}$ centered at $q_{0}^{\prime}$ to a point on the right side, and then along the right side to $p_{i}^{\prime}$, the triangle inequality states

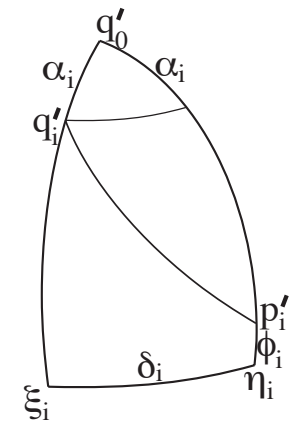

FiguRe 3.

See Figure 3.

$$
\left|q_{i}^{\prime} p_{i}^{\prime}\right| \leq \delta_{i} \sin \alpha_{i}+\left(\left|q_{0}^{\prime} p_{i}^{\prime}\right|-\alpha_{i}\right)
$$

Therefore

$$
\begin{aligned}
\left|q_{i}^{\prime} p_{i}^{\prime}\right|+\phi_{i} & \leq \delta_{i} \alpha_{i}+\left(\left|q_{0}^{\prime} p_{i}^{\prime}\right|-\alpha_{i}\right)+\phi_{i} \\
& =\pi / 2-\alpha_{i}+\delta_{i} \alpha_{i} .
\end{aligned}
$$

In $S_{K}$, extend the model triangle $\widetilde{p}_{0} \widetilde{q}_{0} \widetilde{q}_{i}$ to a quadrilateral $\widetilde{p}_{0} \widetilde{q}_{0} \widetilde{q}_{i} \widetilde{p}_{i}$, by letting $\widetilde{p}_{0} \widetilde{q}_{i} \widetilde{p}_{i}$ be a model triangle for $p_{0} q_{i} p_{i}$ where $\widetilde{p}_{i}$ and $\widetilde{q}_{0}$ lie on opposite sides of $\widetilde{p}_{0} \widetilde{q}_{i}$. Then by (4.9),

$$
\begin{aligned}
\angle \widetilde{q}_{i} \widetilde{p}_{0} \widetilde{p}_{i} \leq \angle q_{i} p_{0} p_{i} & =\left|q_{i}^{\prime} p_{i}^{\prime}\right| \\
& \leq \pi / 2-\alpha_{i}+\delta_{i} \alpha_{i}-\phi_{i} \\
& =\pi / 2-\alpha_{i}\left(1-\delta_{i}\right)-\phi_{i} \\
& \leq \pi / 2-\beta_{i}+\beta_{i} \delta_{i}-\phi_{i} \\
& \leq \pi / 2-\beta_{i}-\widetilde{\phi}_{i}+o\left(t_{i}\right) \\
& =\angle \widetilde{q}_{i} \widetilde{p}_{0} \widetilde{z}_{i}+o\left(t_{i}\right),
\end{aligned}
$$

Here (4.10) follows from (4.8), and from $\beta_{i}=O\left(t_{i}\right), \delta_{i} \rightarrow 0$. (4.11) holds because $\beta_{i}+\angle \widetilde{q}_{i} \widetilde{p}_{0} \widetilde{z}_{i}+\widetilde{\phi}_{i}=\pi / 2$. 
Therefore

$$
h\left(t_{i}\right) \leq\left|q_{i} p_{i}\right|=\left|\widetilde{q}_{i} \widetilde{p}_{i}\right| \leq\left|\widetilde{q}_{i} \widetilde{z}_{i}\right|+o\left(t_{i}^{2}\right),
$$

where the last inequality is by (4.11) and the law of cosines in the model space. By (4.12) and (4.7), $h\left(t_{i}\right) \leq \widetilde{h}\left(t_{i}\right)+o\left(t_{i}^{2}\right)$. Since $h$ and $\widetilde{h}$ have the same values and first derivatives from the right at 0 , the proof of the special case is complete.

General case. Now suppose $K>-A^{2}$, so the model $\widetilde{X}$ is a disk of radius $R(K, A)<\infty$ in $S_{K}$. Set

$$
B_{\partial X}(R(K, A)) \stackrel{\text { def }}{=}\left\{q \in X: \operatorname{dist}_{\partial X}(q)<R(K, A)\right\} .
$$

Let $\tau$ be a geodesic of $X$ with $\tau(0) \in B_{\partial X}(R(K, A))$. The preceding case shows that the distance $h$ from $\tau$ to $\partial X$ satisfies the desired differential inequality on the maximal interval about 0 on which $\tau$ lies in $B_{\partial X}(R(K, A))$.

Define $\widetilde{\tau}$ as in the proof of the preceding case, so the distance $h$ from $\tau$ to $\partial X$ and the distance $\widetilde{h}$ from $\widetilde{\tau}$ to $\partial \widetilde{X}$ have the same initial value and derivative. Since the differential inequality converts infinitesimal support properties into global ones, $h \leq \widetilde{h}$ on any interval about 0 of length $<\pi / \sqrt{K}$ in the intersection of the intervals of definition of $\tau$ and $\widetilde{\tau}$. In particular, since $\widetilde{h}$ vanishes at $t= \pm R(K, A)$, the length of $\tau$ is at most $2 R(K, A)<\pi / \sqrt{K}$. Since $\widetilde{h}<R(K, A)$ if $\widetilde{\tau}$ is not a radial geodesic, it follows that $\tau$ can only leave $B_{\partial X}(R(K, A))$ by running along a geodesic whose parameter is distance to $\partial X$.

Suppose $q_{1}, q_{2} \in X-B_{\partial X}(R(K, A))$. Geodesics $\gamma_{i}$ from the $q_{i}$ to a given $p \in \partial X$ must be minimizers to $\partial X$, and hence they have the same direction orthogonal to $\partial X$ at $p$. Therefore one of the $\gamma_{i}$ must be an extension of the other. But $X$ has dimension at least 2 , since the curvature hypothesis on $\partial X$ cannot be realized in dimension 1. It follows that the open superlevel $h>R(K, A)$ is empty. In particular, $X$ has radius $R(K, A)$, or equivalently, $\mathcal{D}$ is nonnegative as claimed in the statement of Theorem 1.8(a). Moreover, $q_{1}=q_{2}$, so the closed superlevel $h \geq R(K, A)$ consists of a single point at distance $R(K, A)$ from $\partial X$.

Proof of Corollary 1.9. Here we assume $\partial X$ has extrinsic curvature $\geq A>0$ in the base-angle sense at each footpoint, where $K>-A^{2}$. Then part (1) of the corollary is the nonnegativity of $\mathcal{D}$, as proved in Theorem 1.8 (general case). Part (2) is the inequality, length $(\tau) \leq 2 R(K, A)$, in Theorem 1.8 (general case).

Concavity of $\operatorname{dist}_{\partial \widetilde{X}}$ follows from (4.3) and (4.4). Thus dist $_{\partial X}$ is also concave, so the maximum set of dist $\partial X$ is convex. The maximum set consists of a single point, since if $\tau$ is a geodesic with no subsegment realizing distance to the boundary, $\operatorname{dist}_{\partial X} \circ \tau$ is very concave. For any CBB space $X$ and concave function $f$ on $X$, there is a 1-Lipschitz strong deformation retraction of $X$ onto the maximum set of $f[\mathrm{PP}$, also see [L3, Pt2]]. This proves part (3).

Remark 4.5. [CDM2, Corollary 2.4] claims that the conclusion of part (3) of Corollary 1.9 holds when $X$ is nonnegatively curved and $\partial X$ is compact and strictly convex (in the sense that a geodesic can only meet $\partial X$ at an endpoint). For $K \geq 0$, this would be stronger than Corollary 1.9 (3).

Corollary 1.10 states that when $K \geq-A^{2}$ and $X$ has radius $R(K, A)$, then $X$ is isometric to a cone of the following type: 
Definition 4.6. If $Y$ is a metric space, the $K$-cone over $Y$ is the warped product

$$
\text { Cone }_{K}(Y)=[0, \pi / 2 \sqrt{K}] \times \operatorname{sn}_{K}(t) Y .
$$

The vertex of $\operatorname{Cone}_{K}(Y)$ is the equivalence class $q_{0}=\{0\} \times Y$. For $K<0$, the ideal $K$-cone over $Y$ is

$$
\text { Cone }_{K}^{\circ}=\mathbf{R} \times \exp \sqrt{-K} t .
$$

A horoball in Cone $_{K}^{\circ}$ is a subset of the form $(-\infty, a] \times \times_{\exp \sqrt{-K} x} Y$.

Proof of Corollary 1.10. (1) We are assuming $K>-A^{2}$ and the radius of $X$ is $R(K, A)$. By the proof of Theorem 1.8 (general case), there is a single point $q$ at distance $R(K, A)$ from $\partial X$, and any geodesic from $q$ has the distance to $\partial X$ as a geodesic parameter. For any $p \in \partial X$, let $\gamma_{p}$ be a minimizer from $q$ to $p$. Then $\gamma_{p}$ is a minimizer from $q$ to $\partial X$, and since $\gamma_{p}$ is orthogonal to $\partial X$ at $p$, then $\gamma_{p}$ is unique.

For any $r \in B_{\partial X}(R(K, A))$, let $p$ be a footpoint of $r$ on $\partial X$. Since $\gamma_{p}$ is orthogonal to $\partial X, r$ lies on $\gamma_{p}$ and so $p$ is the only footpoint of $r$. Thus there a bijection $\phi$ from $X$ onto the closed ball of radius $R(K, A)$ about $q_{0}$ in $\operatorname{Cone}_{K}(\partial X)$, where

$$
\phi\left(\gamma_{p}(t)\right)=(t, p)
$$

for $0<t \leq R(K, A)$, and $\phi(q)=q_{0}$. The set $t=$ constant is a level of dist $\partial X$, and is orthogonal to the $\gamma_{p}$. Thus to show $\phi$ is an isometry, it suffices to show that for fixed $t$ and $t^{\prime}$, the map $\gamma_{p}(t) \rightarrow \gamma_{p}\left(t^{\prime}\right)$ between the corresponding levels is a homothety, where the magnification factor on intrinsic distances is $\operatorname{sn}_{K}\left(t^{\prime}\right) / \mathrm{sn}_{K}(t)$. We do so by combining triangle comparisons with Theorem 1.8(a).

Consider a triangle $q r_{1} r_{2}$ with $r_{1}, r_{2} \in B_{\partial X}(R(K, A))$ lying on a level, dist $\partial X=$ $C>0$. Two sides of this triangle lie along minimizers $\gamma_{i}$ from $q$ to $p_{i} \in \partial X$. We have

$$
\angle q r_{1} r_{2} \geq \angle \widetilde{q} \widetilde{r}_{1} \widetilde{r}_{2}
$$

where $\widetilde{q r}_{1} \widetilde{r}_{2}$ is the model triangle in $\widetilde{X}$. We will take $\widetilde{q}$ to be the center of $\widetilde{X}$, and write $\widetilde{p}_{i}$ for the footpoint on $\partial \widetilde{X}$ of $\widetilde{r}_{i}$, and $\widetilde{\gamma}_{i}$ for the minimizer from $\widetilde{q}$ to $\widetilde{p}_{i}$. By Theorem 1.8(a), the restriction $h$ of $\operatorname{dist}_{\partial X}$ to the side $r_{1} r_{2}$ satisfies $h \geq \widetilde{h}$, where $\widetilde{h}$ is the restriction of $\operatorname{dist}_{\partial \widetilde{X}}$ to a geodesic in $\widetilde{X}$ having the same length and same endpoint distances from the boundary as $r_{1} r_{2}$. By first variation,

$$
\angle p_{1} r_{1} r_{2} \geq \angle \widetilde{p}_{1} \widetilde{r}_{1} \widetilde{r}_{2}
$$

Since

$$
\angle \widetilde{q} \widetilde{r}_{1} \widetilde{r}_{2}+\angle \widetilde{p}_{1} \widetilde{r}_{1} \widetilde{r}_{2}=\pi, \angle q r_{1} r_{2}+\angle p_{1} r_{1} r_{2}=\pi,
$$

(4.13) and (4.14) imply

$$
\angle q r_{1} r_{2}=\angle \widetilde{q} \widetilde{r}_{1} \widetilde{r}_{2}, \angle p_{1} r_{1} r_{2}=\angle \widetilde{p}_{1} \widetilde{r}_{1} \widetilde{r}_{2}
$$

The same equations hold with the indices reversed.

Set

$$
L(t)=\left|\gamma_{1}(t) \gamma_{2}(t)\right|, \quad \widetilde{L}(t)=\left|\widetilde{\gamma}_{1}(t) \widetilde{\gamma}_{2}(t)\right|, \quad 0 \leq t \leq R(K, A) .
$$

By first variation, (4.15) implies $d L / d t=d \widetilde{L} / d t$. Since $L(0)=\widetilde{L}(0)=0$, then $L=\widetilde{L}$.

Thus there is a constant ratio, independent of the particular choice of the $p_{i}$, between an "input" value $L(t)$ and an "output" value $L\left(t^{\prime}\right)$, depending only on $t$, $t^{\prime}$ and the input value. It follows, from the definition of intrinsic distance in terms 
of arclength, and the definition of arclength in terms of broken chord length, that the corresponding levels are homothetic with magnification factor

$$
\lim _{\widetilde{L}(t) \rightarrow 0}\left(\widetilde{L}\left(t^{\prime}\right) / \widetilde{L}(t)\right)=\operatorname{sn}_{K}\left(t^{\prime}\right) / \operatorname{sn}_{K}(t) .
$$

(2) Suppose $K=-A^{2}$ and the radius of $X$ is infinite. Recall that $\widetilde{X}$ is a horodisk in the hyperbolic plane of curvature $K$.

A ray starting at any point $r \in X$ may be obtained in the limit, from a sequence of minimizers from $r$ to points whose distance from $r$ increases without bound. As in the proof of Theorem 1.8 (general case), any ray has the distance to $\partial X$ as a geodesic parameter because $\widetilde{X}$ has the same property. Then just as in part (a) of this corollary, there is a unique ray $\gamma_{p}$ from any $p \in \partial X$, and it is orthogonal to $\partial X$. Moreover, a ray from any $r \in X$ is unique and runs along $\gamma_{p}$, where $p$ is the unique footpoint of $r$ on $\partial X$.

Consider $r_{1}, r_{2}$ on a level $\operatorname{dist}_{\partial X}=C>0$, and let $\gamma_{i}$ be the ray from $p_{i} \in \partial X$ through $r_{i}$. Choose $\widetilde{r}_{1}, \widetilde{r}_{2}$ in $\widetilde{X}$ on the level $\operatorname{dist}_{\partial \widetilde{X}}=C$, and satisfying $\left|r_{1} r_{2}\right|=$ $\left|\widetilde{r}_{1} \widetilde{r}_{2}\right|$. Let $\widetilde{\gamma}_{i}$ be the ray from $\widetilde{p}_{i} \in \partial \widetilde{X}$ through $\widetilde{r}_{i}$.

Let $q_{i}$ lie on the ray $\gamma_{i}$ and on a level dist $\partial X=C^{\prime}>C$, and let $\widetilde{q}_{i}$ lie on $\widetilde{\gamma}_{i}$ and on the level $\operatorname{dist}_{\partial \widetilde{X}}=C^{\prime}$. We must verify that the inequality analogous to (4.13) holds, namely

$$
\angle q_{1} r_{1} r_{2} \geq \angle \widetilde{q_{1}} \widetilde{r}_{1} \widetilde{r}_{2}
$$

First note that dist $_{\partial X}$ is concave. Indeed, the differential inequality of Theorem 1.8 (b) trivially reduces to the differential inequality

$$
f^{\prime \prime} \leq-\sqrt{-K}\left(1-\left(f^{\prime}\right)^{2}\right) \leq 0
$$

on $f=\operatorname{dist}_{\partial X}$. Therefore the superlevel $\operatorname{dist}_{\partial X}>C^{\prime}$ is convex, so as $C^{\prime} \rightarrow \infty$, the points on any geodesic $\tau_{C^{\prime}}$ joining $q_{1}$ and $q_{2}$ move arbitrarily far from the $r_{i}$. Since $\left|r_{1} q_{1}\right|<\left|r_{1} q_{2}\right|$ and $\left|r_{2} q_{2}\right|<\left|r_{2} q_{1}\right|$, by the uniqueness of the $\gamma_{i}$, then there is a point $q$ on $\tau_{C^{\prime}}$ at which $\left|r_{1} q\right|=\left|r_{2} q\right|$. Now we have

$$
\angle q r_{1} r_{2} \geq \angle \widetilde{q} \widetilde{r}_{1} \widetilde{r}_{2}
$$

where $\widetilde{q} \widetilde{r}_{1} \widetilde{r}_{2}$ is a model isosceles triangle for $q r_{1} r_{2}$. As $C^{\prime} \rightarrow \infty$, the sides $\widetilde{r}_{i} \widetilde{q}$ converge to subrays of the $\widetilde{\gamma}_{i}$, and by the uniqueness of the ray from $r_{i}$, the sides $\left|r_{i} q\right|$ converge to subrays of the $\gamma_{i}$. (4.16) follows. The remainder of the proof is identical to that of part (a).

\section{BASE-ANGLE/ChORD AND ARC/ChORD CURVATURE BOUndS}

In this section we show that a uniform lower bound on extrinsic curvature of the boundary of a CBB space $X$, in the base-angle/chord sense similar to (4.2), implies a uniform arc/chord form, as in Definition 3.1. However, the version (4.2) is pointwise with the error terms given in terms of chord lengths $r$, while the conclusion we want is uniform with the error terms given in terms of arclength $s$. So we have to formulate the hypothesis uniformly in terms of arclength, as follows.

Definition 5.1. An intrinsically geodesic subset $Y$ of a CBA or CBB space $X$ is a subset of $X$ that is geodesic in its intrinsic metric, and whose geodesics have one-sided directions in $X$. An intrinsically geodesic subset $Y$ has base-angle/chord curvature $\geq A$ if there is a function $F(s) \geq 0$ satisfying $\lim _{s \rightarrow 0}(F(s) / s)=0$, such 
that for every intrinsic geodesic $\tau$ in $Y$ of length $s<s_{0}$ and chord $\sigma$ in $X$ between the endpoints of $\tau$, the angles $\alpha$ between $\tau$ and $\sigma$ satisfy

$$
\alpha \geq A s / 2-F(s) .
$$

If we only consider endpoints in an open subset $Z \subset Y$, we say $Y$ has baseangle/chord curvature $\geq A$ on $Z$.

Examples of intrinsically geodesic subsets include extremal subsets of CBB spaces $X$, and in particular, boundaries $\partial X$ of finite-dimensional CBB spaces $X[\mathrm{Pt} 2,4.1]$. Any curve with one-sided directions is an intrinsically geodesic subset.

Remark 5.2. It is straightforward to verify that Definition 5.1 is equivalent to the following comparison version: For every $0<A^{\prime}<A$, there is $s\left(A^{\prime}\right)>0$ such that for every intrinsic geodesic ("arc") in $Y$ of length $s<s\left(A^{\prime}\right)$ and chord between its endpoints, the angles $\alpha$ between arc and chord satisfy

$$
\alpha \geq A^{\prime} s / 2 \text {. }
$$

In the proof of Theorem 5.3 we will use the comparison versions of Definition 5.1 and Definition 3.1.

Theorem 5.3. If an intrinsically geodesic subset $Y$ of a $C B A$ or $C B B$ space $X$ has base-angle/chord curvature $\geq A>0$ on an open subset $Z \subset Y$, then $Y$ has arc/chord curvature $\geq A$ on $Z$.

Proof. We consider a geodesic $\operatorname{arc} \sigma$ in $Y$, with fixed initial point $\sigma(0)=p$. Then the chord length $r=|p \sigma(s)|$ is a function of $s$ which, by first variation, has a left derivative at every point and has right derivative 1 at $s=0$, since $\sigma$ has a direction in $X$ there: $s-r=o(s)$.

Specifically, the first variation formula tells us that the left derivative of $r$ is given by $r_{-}^{\prime}(s)=\cos \left(\alpha_{m}(s)\right)$, where $\alpha_{m}(s)$ is the minimum of the angles which the various chords from $p$ to $\gamma(s)$ make with the left direction of $\sigma$. Our hypothesis gives that for every $0<A^{\prime}<A$ there is $s\left(A^{\prime}\right)>0$ such that for $0<s<s\left(A^{\prime}\right)$, $\alpha_{m}(s)>A^{\prime} s / 2$. Hence $r_{-}^{\prime}(s)<\cos \left(A^{\prime} s / 2\right)$. The constants $s\left(A^{\prime}\right)$ can be chosen to depend only on $F$ and may be assumed to satisfy $\lim _{A^{\prime} \rightarrow A} s\left(A^{\prime}\right)=0$.

In turn, for $0<s<s\left(A^{\prime}\right)$,

$$
\begin{aligned}
\frac{d s}{d s}-r_{-}^{\prime}(s)>1-\cos \left(\frac{A^{\prime}}{2} s\right) & =\frac{\left(A^{\prime}\right)^{2}}{8} s^{2}-\cos \left(\frac{A^{\prime}}{2} s_{1}\right) \frac{\left(A^{\prime}\right)^{4}}{384} s^{4} \\
& >\frac{\left(A^{\prime}\right)^{2}}{8} s^{2}\left(1-\frac{\left(A^{\prime}\right)^{2}}{48} s\left(A^{\prime}\right)^{2}\right),
\end{aligned}
$$

for some $0<s_{1}<s$. Then there is a continuous function

$$
f(s)=s-r(s)-\frac{\left(A^{\prime}\right)^{2}}{24} s^{3}\left(1-\frac{\left(A^{\prime}\right)^{2}}{48} s\left(A^{\prime}\right)^{2}\right)
$$

with positive left derivative everywhere in the open interval $\left(0, s\left(A^{\prime}\right)\right)$. Hence $f(s)$ has no minimum point in that interval, and since $f(0)=0$, then $f(s)>0$ in that interval. That is,

$$
s-r>\frac{\left(A^{\prime}\right)^{2}}{24}\left(1-\frac{\left(A^{\prime}\right)^{2}}{48} s\left(A^{\prime}\right)^{2}\right) s^{3}
$$

if $s<s\left(A^{\prime}\right)$. Since $\lim _{A^{\prime} \rightarrow A} s\left(A^{\prime}\right)=0$, the claim follows. 
Corollary 5.4. If $X$ is a finite-dimensional $C B B$ space with $\partial X \neq \emptyset$, and $\partial X$ has base-angle/chord curvature $\geq A$ on an open subset $Z \subset \partial X$, then $\partial X$ has arc/chord curvature $\geq A$ on $Z$.

\section{ACKNOWLEDGMENTS}

In June 2007, after obtaining Theorems 1.3, 1.6 and 1.8, we learned that Jianguo Cao, Bo Dai and Jiaqiang Mei had been doing related work independently [CDM1]. We thank Jianguo Cao for discussions of this work. It appears to us that the claim made there about deriving global topological information from positive curvature in the neighborhood of a point is a challenging one, and proving it would require arguments that go beyond those in this paper or in [CDM1].

\section{REFERENCES}

[AB1] S. Alexander, R. Bishop, Comparison Theorems for Curves of Bounded Geodesic Curvature in Metric Spaces of Curvature Bounded Above, Differential Geometry and its Applications, 6 (1996), 67-86.

[AB2], $\mathcal{F} K$-convex functions on metric spaces, Manuscripta Math. 110:1, (2003), 115133. MR 2004a:53100

[AB3] Curvature bounds for warped products of metric spaces, Geom. Funct. Anal. 14 (2004), 1143-1181.

[AB4] Gauss Equation and Injectivity Radii for Subspaces in Spaces of Curvature Bounded Above, Geom.Dedicata, 117 (2006), 65-84.

[A] A. D. Alexandrov, Ruled surfaces in metric spaces, Vestnik Leningrad. Univ., 12:5-26, 1957 (Russian).

[Ba] W. Ballmann, Lectures on Spaces of Nonpositive Curvature. Basel, Boston: BirkhauserVerlag, 1995; DMV Seminar Bd. 25.

[BN] V. N. Berestovskii, I. G. Nikolaev, Multidimensional generalized Riemannian spaces, in Geometry IV. Non-regular Riemannian Geometry. Encyclopaedia of Math. Sciences Springer-Verlag, Berlin, Heidelberg, 1993, 165-244.

[BH] M. Bridson, A. Haefliger, Metric Spaces of Non-positive Curvature, Springer-Verlag, 1999.

[BBI] D. Burago, Yu. Burago, S. Ivanov, A Course in Metric Geometry, Graduate Studies in Mathematics, Vol. 33, Amer. Math. Soc., Providence, 2001.

[CDM1] J. Cao, B. Dai, J. Mei, An extension of Perelman's soul theorem for singular spaces, arXiv:0706.0565v1, v2(19 June 2007), v3(19 Dec. 2007).

[CDM2] _ An optimal extension of Perelman's comparison theorem for quadrangles and its applications, arXiv:0712.3221v1(19 Dec. 2007).

[D] B. Dekster, Estimates of the length of a curve, J. Differential Geometry, 12 (1977), 101-118.

[KPT] V. Kapovitch, A. Petrunin, W. Tuschmann, Nilpotency, almost nonnegative curvature and the gradient flow, Annals of Math., to appear.

[L1] A. Lytchak, Geometry of Sets of Positive Reach, Manuscripta Math., 115 (2004), 199205.

[L2] _ Almost Convex Subsets, Geom. Dedicata, 115 (2005), 201-218.

[L3] _ Open Map Theorem for Metric Spaces, Algebra i Analiz, Vol.17 (2005), 139-159, St. Petersburg Math. J.

[M] C. Mese, The curvature of minimal surfaces in singular spaces, Comm. Anal. Geom. 9 (2001), 3-34.

[Pr] G. Perelman, Alexandrov's spaces with curvature bounded from below II, preprint (1991).

[PP] G. Perelman, A. Petrunin, Quasigeodesics and Gradient Curves in Alexandrov Spaces, preprint (1994), www.math.psu.edu/petrunin.

[Pt1] A. Petrunin, Metric minimizing surfaces, Elec. Res. Announc. Amer. Math. Soc. 5 (1998), 47-54.

[Pt2] Semiconcave functions in Alexandrov's geometry, Surveys in Differential Geometry, 11, Int. Press, Somerville, MA, (2007), 137-201. 
[Pl] C. Plaut, Metric spaces of curvature $\geq k$. R. Daverman, R. Sher (eds.), Handbook of Geometric Topology, Elsevier, (2002), 819-898.

1409 W. Green St., Urbana, IL 61801

E-mail address: sba@math.uiuc.edu

1409 W. Green St., Urbana, IL 61801

E-mail address: bishop@math.uiuc.edu 